\title{
ASYMPTOTIC APPROXIMATIONS TO THE NON-ISOTHERMAL DISTRIBUTED ACTIVATION ENERGY MODEL FOR BIO-MASS PYROLYSIS
}

\author{
Alok DHAUNDIYAL*, Suraj B. SINGH ** \\ ${ }^{*}$ Faculty of Mechanical Engineering, Mechanical Engineering PhD School, Szent Istvan University, Godollo, Hungary \\ ** Department of Mathematics, Statistics and Computer Science, Govind Ballabh Pant University of Agriculture and Technology, \\ Pantnagar, Uttarakhand 263153, India
}

Dhaundiyal.Alok@phd.uni-szie.hu, drsurajbsingh@yahoo.com

received 17 June 2016, revised 23 November 2017, accepted 28 November 2017

\begin{abstract}
This paper describes the influence of some parameters significant to biomass pyrolysis on the numerical solutions of the non-isothermal nth order distributed activation energy model (DAEM) using the Gamma distribution and discusses the special case for the positive integer value of the scale parameter $(\lambda)$, i.e. the Erlang distribution. Investigated parameters are the integral upper limit, the frequency factor, the heating rate, the reaction order, and the shape and rate parameters of the Gamma distribution. Influence of these parameters has been considered for the determination of the kinetic parameters of the non-isothermal nth order Gamma distribution from the experimentally derived thermoanalytical data of biomass pyrolysis. Mathematically, the effect of parameters on numerical solution is also used for predicting the behaviour of the unpyrolysized fraction of biomass with respect to temperature. Analysis of the mathematical model is based upon asymptotic expansions, which leads to the systematic methods for efficient way to determine the accurate approximations. The proposed method, therefore, provides a rapid and highly effective way for estimating the kinetic parameters and the distribution of activation energies.
\end{abstract}

Key words: Biomass Pyrolysis, Distributed Activation Energy Model (DAEM), Non- Isothermal Kinetics, Gamma Distribution, Asymptotic Expansion, Erlang Distribution

\section{INTRODUCTION}

There are various possible ways of converting biomass into valuable products (Szczodrak and Fiedurek, 1996). One of them is pyrolysis, which comes under purview of thermochemical processes. While biomass undergoes pyrolysis process, it changes into gases, liquid oil and char. The details studies related to pyrolysis and thermal analysis is reported by Colomba Di Blasi (Blasi, 2008) and John E White (White and Legendre, 2011). Thermogravimetric analysis (TGA) is mainly used for experimental observation of pyrolysis decomposition (Brown, 2001). The main objective of thermogravimetric analysis is to measure changes of mass as functions of time or temperature.

Mathematically, it becomes complicated to describe biomass decomposition kinetics, as several reactions take place and their mechanism is unknown. There are various models which have been used to explain biomass pyrolysis, such as single-reaction and multi-reaction models (Carpart et al., 2004; Conesa et al., 1995; Conesa et al, 2001; Pysiak and Badwi, 2004; Mysyk et al. 2005; CriadoJ Pérez-Maqueda, 2005). The most common approach used is isoconversional model, which assumes that kinetic parameters, such as the frequency factor and activation energies, are not constant during the process of decomposition. Another model, which is also used for determination of kinetic parameters, is the lumped kinetics model. It postulates that an ultimate number of parallel $\mathrm{n}^{\text {th }}$ order reactions take place. These partial reactions provide the information about overall decomposition run. Howev- er, hitherto, the most accurate and up-to-date approach has been implemented for the modeling of biomass pyrolysis is the distributed activation energy model (DAEM) (Burnham and Braun, 1999; Burnham et al.,1995; Galgano and Blasi, 2003; Ferdous et al., 2002). Comparatively, the principle of the lumped kinetic model is very similar to the DAEM. The only difference is in the number of expected decomposition reactions. However, lumped kinetic model contains around one-hundred decomposition reactions, it would be approaching the distributed activation kinetic model.

The numerical solutions obtained, after implementing the asymptotic expansion, of the kinetic model are used to determine the kinetic parameters. To predict realistic results, the parameters affecting the behaviour of numerical solution must be estimated. The effect of these parameters on the single -reaction models has been reviewed in literature (Brown, 2001). The distributed activation energy model (DAEM) has proven very successful to describe the pyrolysis of different types of biomass. Our study mainly focuses on the relevant parameters which affect the kinetics of pyrolysis. The DAEM also applies to the pyrolysis of other conventional sources of energy like coal, residual oils, resin chars (Teng and Hsieh, 1999) and kerogen (Lakshmanan and White, 1994). This analytical method is not only used for thermal decomposition of plant, or animal biomass (Giuntoli et al., 2009; Lapuerta et al., 2004), but also for thermal decomposition of other materials such as medical wastes (Zhu et al., 2009), waste car tyres ([Koreňová et al., 2006), printed circuit board wastes (Quan et al., 2009), or sewage sludge (Otero et al. 2008; Folgueras et al. 2003). Approximation to the non-isothermal distribution requires 
many evaluations of double exponential term (DExp) which involves rapidly varying functions, and this leads to significant numerical difficulties. In addition to that the double exponential term arising in the DAEM, is investigated for the non-isothermal temperature profile only. This double exponential term acts over a narrow range of activation energies, which varies as time proceeds. While approximating, the significant part of our analysis is to identify the importance of relative step width of the double exponential term as compared to the width of the initial distribution of volatiles released during pyrolysis. The main aim of this study is to use asymptotic methods to make accurate approximation to the integrals, and thereby predict the behaviour of the non-isothermal nth order DAEM by considering the effect of some relevant parameters on the pyrolysis of biomass and discuss the behaviour of distribution for the special case (Erlang distribution).

\section{THE NON-ISOTHERMAL NTH ORDER DAEM USING THE GAMMA DISTRIBUTION AND ITS ASYMPTOTIC APPROXIMATION}

The concept of distributed activation energy was originally propounded by Vand (1943). Pitt applied DAEM to the problem of coal devolatilization (Pitt, 1962). Later, it was used by Hanbaba and his co-workers (Hanbaba, 1968), and Anthony (Anthony, 1974). Postulation of the model states that the decomposition mechanism takes many independent, parallel, first order chemical reactions with different activation energies which reflect variations in the bond strength of constituent of biomass. The development of the model here follows Howard (Howard,1981) and Solomon and Hamblen (Solomon and Hamblen, 1983). Assume biomass's constituents are numbered $i=1,2,3 \ldots \ldots n$, and the released (volatilized mass fraction for the $i^{\text {th }}$ constituent is $V_{i}(t)$. The initial mass of constituent $i$ in the coal is $V_{i}^{*}$. Each reaction is assumed to be first order, so that the rate of pyrolysis is given by equation (1)

$$
\frac{d V_{i}}{d t}=k_{i}\left(V_{i}^{*}-V_{i}\right)
$$

The rate coefficient is taken to be Arrhenius in the form

$k_{i}=k_{0 i} e^{-\frac{E_{i}}{R T}}$,

where $\mathrm{k}_{0 \mathrm{i}}$ is the pre-exponential or frequency factor $\left(s^{-1}\right), E_{i}$ is the apparent activation energy for constituent $i(\mathrm{~kJ} / \mathrm{mol}), R$ is ideal gas constant, and $T(t)$ is the time-dependent temperature $(\mathrm{K})$ regime of the biomass.

Equation (1) can be written as:

$\left(V_{i}^{*}-V_{i}\right)=e^{-\int_{0}^{t} k_{i} d t}$

For $\mathrm{i}=1$, the model is referred to as the single first-order reaction model (SFOR). In DAEM, the dependence on volatile number $\mathrm{i}$ is substituted by a continuous distribution function of activation energy $\mathrm{E}$, so that the total fraction of volatile available to release from biomass is considered to follow distribution correctly (Equation (2)).

$d V^{*}=f(E) d E$

The solution then becomes:

$\frac{V^{*}-V}{V^{*}}=\int_{0}^{\infty} \exp \left(-\int_{0}^{t} k_{0}(E) e^{-\frac{E}{R T}}\right) f(E) d E$
The main purpose of using this model is to assume the initial distribution of volatile $f(E)$, the pre-exponential factors $k_{0}(E)$, and then find the resulting time-dependence of the volatiles. This model involves reaction time scale, which gained lot of acceptance as it is most significant part of biomass devolatilization (Howard, 1981; Suuberg, 1983). The problem relating to DAEM is that the function $f(E)$ and $k_{0}(E)$ are highly correlated, hence it is very complicated for us to determine both functions accurately. A common assumption has been considered to assume the constant value of pre-exponential or frequency factors $k_{0}$. By doing so, though analysis gets easier, but study is more focused towards the uncertainty of reactant distributions. In this paper, approximation for the time-dependence of the volatiles is evaluated first, which is given by equation (2), where $k_{0}(E)$ is replaced by the constant $k_{0}$. The non-isothermal nth DAEM is given by equation (3)

$1-X=$

$\left\{\begin{array}{l}\int_{0}^{\infty} \exp \left[-\int_{0}^{t} k_{0} \exp \left(\frac{-E}{R T}\right) d t\right] f(E) d E \text { (first order reaction) } \\ \int_{0}^{\infty}\left[1-\left(1-n \int_{0}^{t} k_{0} \exp \left(\frac{-E}{R T}\right) d t\right)\right]^{\left(\frac{1}{1-n}\right)} f(E) d E \text { for } n \neq 1\end{array}\right.$

where $(1-\mathrm{X})$ is the mass fraction of released volatile, $n$ is the order of reaction, and $f(E)$ is the distribution of activation energies.

Mainly, $f(E)$ is a Gaussian distribution function. As the selection of an appropriate distribution function for the molecular activation energies is very important component of our study, so it would be beneficial to select an asymmetric distribution for modeling the kinetics of biomass pyrolysis, such as the Gamma distribution, over a symmetrical one (Skrdla and Roberson, 2005).

Moreover, the Gamma distribution is mathematically flexible and expressed as:

$f(E)=\frac{E^{(\lambda-1)} e^{-\frac{E}{\eta}}}{\eta^{\lambda} \Gamma(\lambda)}$ for $E>0$

where $\lambda$ is positive scale parameter expressed in $\mathrm{kJ} / \mathrm{mol}$ and, $\eta$ is dimensionless positive shape parameter.

The mean and the variance of distribution are given by equation (5) and equation (6) respectively:

$E_{0}=\frac{\lambda}{\eta}$

$\sigma^{2}=\frac{\lambda}{\eta^{2}}$

Primarily, the Erlang distribution was given by A. K. Erlang to examine the telephonic-traffic engineering. Later, it was implemented in the field of stochastic and biomathematics (Robeva, 2010). Basically, the Erlang distribution is a special case of the Gamma distribution for the integer values of the scale parameter $\lambda$. For $\eta=1$, the distribution is exponential. For values of $\eta \geq 1$, the distribution becomes 'bell shaped', and positively skewed. With increase in the value of $\eta$, the Gamma distribution approaches the Gaussian distribution more and more closely. In addition, attribute of distribution curve is also decided by the scale and shape parameters. However, the wide distribution pattern is chosen to simplify the given numerical problem of DAEM. Here, variance and mean of distribution functions are dependent on these parameters, which are evaluated with the help of equations (5) and (6). 


\section{SYSTEMATIC APPROXIMATIONS}

The integrand in equation (3) (first order) consists of two terms. The first term (DExp) of equation (3) is function of time, which in fact depends on the temperature history experienced experimentally by the biomass sample. The second part is invariant of time, and depends on the distribution of volatiles in the sample. The behaviour of temperature dependent part is considered first, and approximations are derived that is useful to solve the double exponential term. The ramping temperature history only has been investigated, together with the Gamma distribution of volatiles.

\subsection{The double exponential simplification}

Approximations to the double exponential are considered, where $T$ varies linearly with time and having constant slope that indicates the heating rate $(\theta)$ of sample $E$ can take any positive value. The approach considered here is similar to that of Niksa and Lau (Niksa and Lau, 1993) and it involves much systematic and accurate approximation.

$\operatorname{DExp}=\exp \left(-\int_{0}^{t} k_{0} e^{-\frac{E}{R T}} d t\right)$

For approximation of double exponential term, the first step is to consider the typical values of dependent parameters and functions. The frequency factors $\left(\mathrm{k}_{0}\right)$ are typically in range of $\mathrm{k}_{0} \sim 10^{10}-10^{13} \mathrm{~s}^{-1}$, whereas the activation energies domain lies between 100-300 $\mathrm{kJ} / \mathrm{mol}$. The temperature dependence, however, consider according to the specified experimental requirement. It varies from biomass to biomass, but typically the range of temperature varies as:

$1000{ }^{\circ} \mathrm{C} \leq T \leq 2000{ }^{\circ} \mathrm{C}$

Note: The DAEM model is also applicable to the combustion related problems where the extensive range of temperature is incorporated, and hence it is useful to extrapolate the simplification made in the higher specified regimes of temperature, which is mentioned above.

To demonstrate the proposed method, the ramping profile of temperature has been considered as follows

$\mathrm{T}=\theta \mathrm{t}$,

If the temperature is taken to ramp linearly, DExp becomes:

$\operatorname{DExp}=\exp \left(-\int_{0}^{t} k_{0} e^{-\frac{E}{R \theta t}} d t\right)$

The integral in the exponent is approximated by using the conventional Laplace approach where the parameter $\frac{\mathrm{E}}{\mathrm{R} \theta \mathrm{t}}$ is assumed to be large and hence the dominant contribution from the integral is nearest to the maximum temperature experienced by the biomass sample.

$$
\exp \left(-\int_{0}^{t} k_{0} e^{-\frac{E}{R \theta t}} d t\right) \sim \exp \left(\frac{-k_{0} R \theta t^{2}}{E} e^{-\frac{E}{R \theta t}}\right)
$$

as $\frac{E}{R \theta t} \rightarrow \infty$
This approximate function can be written in the form:

$\sim \exp \left(-\exp \left(\frac{E_{s}-E}{E_{w}}\right)\right)$

where again the function switches rapidly from zero to one with respect to increment of activation energy $E$, over a range of step size $E_{w}$ around the central value $E_{s}$, which can be approximated as follows:

$g(E)=\left(\frac{E_{s}-E}{E_{w}}\right)$

Then equation (7) can be rewritten as:

$\exp (-\exp (g(E)))$

where:

$g(E) \equiv-\frac{E}{R \theta t}+\ln \left(\frac{k_{0} R \theta t^{2}}{E}\right)$

Expand $g(E)$ with the help of Taylor series around $E_{s}$ by:

$g(E) \sim g\left(E_{S}\right)+\left(E-E_{S}\right) g^{\prime}\left(E_{S}\right)+\frac{\left(E-E_{S}\right)^{2}}{2 !} g^{\prime \prime}\left(E_{S}\right)+$

Using equation (8) and the predefined function $\mathrm{g}\left(\mathrm{E}_{\mathrm{s}}\right), \mathrm{E}_{\mathrm{s}}$ and $\mathrm{E}_{\mathrm{w}}$ are chosen such that:

$g\left(E_{\mathrm{S}}\right)=0$ and $g^{\prime}\left(E_{\mathrm{s}}\right)=\frac{-1}{E_{\mathrm{w}}}$

After solving these, we have:

$E_{s}=R \theta t Y\left(k_{0} t\right)$ and $E_{w}=\left(\frac{R \theta t E_{S}}{R \theta t+E_{s}}\right)$

where $\mathrm{Y}(\mathrm{x})$ is Lambert $\mathrm{W}$ function defined to be one of the real roots of the equation:

$Y e^{Y}=x$.

Approximation for the small and the large values of $x$ (corresponding to short and long times) (Armstrong and Kulesza, 1981) can be taken as:

$Y \sim x-x^{2}, x \ll 1$,

and

$Y \sim \ln \left(\frac{x}{\ln \left(\frac{x}{\ln x}\right)}\right), x \gg 1$.

DExp has been varied like a smoothed step-function, rising rapidly (for the large values of $k_{0} t$ ) from zero to one in a range of activation energies of the step width $E_{w}$ around the central value $E=E_{s}$, where both $E_{s}$ and $E_{w}$ vary with time. In equation (3), DExp is multiplied by the initial distribution $f(E)$. The initial distribution is supposed to be centered around a value E0 and has a width designated by $\sigma$. The Gamma distribution function has been used, which is demarcated by the special case, i.e the $\mathrm{Er}$ lang distribution for its practical scope. The distribution can be either wide or narrow. Here we have discussed the wide distribution case only, where the initial distribution function $f(E)$ is relatively wide in compare with the width of DExp. Moreover, the 
shape of the total integrand also depends on the distribution chosen. When the initial distribution is relatively wide compared to $\mathrm{E}_{\mathrm{w}}$, the total integrand behaves similar to an initial distribution $\mathrm{f}(\mathrm{E})$. But as time proceeds, it is progressively shifted from the left by the step-like DExp. The location of the maximum of the total integrand can move significantly, and the shape becomes quite skewed.

From equations (3) and (7), the remaining mass fraction equation can be expressed as:

$1-X=\int_{0}^{\infty} \exp \left(-\exp \left(\frac{E_{s}-E}{E_{w}}\right)\right) \frac{E^{(\lambda-1)} e^{-\frac{E}{\eta}}}{\eta^{\lambda} \Gamma(\lambda)} d E$

Let:

$h(E)=-\exp \left(\frac{E_{S}-E}{E_{w}}\right)-\frac{E}{\eta}$,

then:

$1-X=\int_{0}^{\infty} \exp (h(E)) \frac{E^{(\lambda-1)}}{\eta^{\lambda} \Gamma(\lambda)} d E$

where $E_{S}$ and $E_{w}$ are function of $t$ as mentioned earlier.

Energy is now rescaled as $y=\frac{E}{E_{0}}$, so that the problem becomes:

$1-X=\frac{\alpha}{\Gamma(\lambda)} \int_{0}^{\infty} y^{(\lambda-1)} \exp (h(y)) d y$

$h(y)=-\exp \left(\frac{y_{s}-y}{y_{w}}\right)-(\sigma \sqrt{y})^{2}$

where the constant parameter $\alpha=\frac{\sigma^{2 \lambda}}{\mathrm{E}_{0}}$. Note that in practice $\alpha \ll 1$. Time is also rescaled as $\tau=\mathrm{k}_{0} \mathrm{t}$.

For linear ramping temperature $\mathrm{T}=\theta \mathrm{t}$,

$y_{s}=\frac{R \theta \tau Y(\tau)}{k_{0} E_{0}}, y_{w}=\frac{y_{s}}{(1+Y(\tau))}$

Note: The ramping temperature can be generalized to the case of non-zero initial temperature $T_{0}$ by simply replacing $t$ with $\mathrm{t}+\frac{\mathrm{T}_{0}}{\theta}$ everywhere, else analysis will be changed.

Approximations to equation (10) are studied by considering the initial distribution, centered around $\mathrm{y}=1$ with width $\sqrt[2 \lambda]{\alpha}$, while DExp jumps from zero to one around $y=y_{s}$ with a width $\mathrm{y}_{\mathrm{w}}$.

\subsection{The wide distribution case}

\subsubsection{Gamma distribution (Generalized form of the Erlang distribution)}

The initial distribution much wider than DExp is considered. In this limit, as previously discussed, DExp jumps from zero to one near $\mathrm{y}=\mathrm{y}_{\mathrm{s}}$ in a manner that has previously been approximated by the step function (Howard, 1981; Suuberg, 1983; Vand, 1943; Pitt, 1962). To apply this, the limit $\mathrm{y}_{\mathrm{w}} \sqrt[2 \lambda]{\alpha} \ll 1$ is taken:

$H\left(y-y_{s}\right)=\left\{\begin{array}{l}0, y<y_{s} \\ 1, y \geq y_{s}\end{array}\right.$
Equation (9) can be rewritten for first order and $\mathrm{n}^{\text {th }}$ order reactions

\section{Case 1: For first order $(\mathbf{n}=\mathbf{1})$ reaction}

$1-X=$

$\frac{\alpha}{\Gamma(\lambda)} \int_{0}^{\infty}\left(\exp \left(-\exp \left(\frac{y_{s}-y}{y_{w}}\right)\right)-\right.$

$\left.H\left(y-y_{s}\right)\right) y^{(\lambda-1)} \exp \left(-(\sigma \sqrt{y})^{2}\right) d y+\frac{\alpha}{\sigma^{2 \lambda} \Gamma(\lambda)} \Gamma\left(\lambda, \sigma^{2} y_{s}\right)$

where $\Gamma\left(\lambda, \sigma^{2} y_{s}\right)$ is the upper incomplete Gamma function.

The second integral in this equation is a complementary error function, therefore easily computed. In fact, many previous simplifications (the step-function approximations) used just this term and neglected the first integral. The first integral term is the initial distribution multiplied by a function that is very small everywhere except in a neighbourhood of size $\mathrm{y}_{\mathrm{w}}$ around the point $\mathrm{y}=\mathrm{y}_{\mathrm{s}}$. This integrand can, therefore, be approximated by expanding the initial distribution term with the help of Taylor series about $y=y_{s}$.

Let:

$$
\begin{aligned}
& Z(y)=y^{(\lambda-1)} \exp \left(-(\sigma \sqrt{y})^{2}\right) \\
& Z(y) \sim Z\left(y_{s}\right)+\left(y-y_{s}\right) Z^{\prime}\left(y_{s}\right)+\frac{\left(y-y_{s}\right)^{2}}{2 !} Z^{\prime \prime}\left(y_{s}\right)+ \\
& \frac{\left(y-y_{s}\right)^{3}}{3 !} Z^{\prime \prime \prime}\left(y_{s}\right)+\ldots \\
& Z(y) \sim Z\left(y_{s}\right)+\left(y-y_{s}\right) Z^{\prime}\left(y_{s}\right)+\frac{\left(y-y_{s}\right)^{2}}{2 !} Z^{\prime \prime}\left(y_{s}\right)+\frac{\left(y-y_{s}\right)^{3}}{3 !} Z^{\prime \prime \prime}\left(y_{s}\right) \\
& \sim \exp \left(-\left(\sigma \sqrt{y_{s}}\right)^{2}\right)\left[y_{s}{ }^{(\lambda-1)}-\left(y-y_{s}\right) \mathrm{y}_{s}{ }^{(\lambda-2)}\left(-\lambda+\sigma^{2} y_{s}+1\right)+\right. \\
& \frac{\left(y-y_{s}\right)^{2}}{2} y_{s}(\lambda-3)\left(\sigma^{4} y_{s}^{2}+2(1-\lambda) \sigma^{2} y_{s}+\left(\lambda^{2}-3 \lambda+2\right)\right)- \\
& \frac{\left(y-y_{s}\right)^{3}}{6} y_{s}{ }^{(\lambda-4)}\left(\sigma^{6} y_{s}{ }^{3}+3(1-\lambda) \sigma^{4} y_{s}{ }^{2}+\left(3 \lambda^{2}-9 \lambda+6\right) \sigma^{2} y_{s}-\right. \\
& \left.\left.\lambda^{3}+6 \lambda^{2}-11 \lambda+6\right)\right]
\end{aligned}
$$

Substituting $\frac{\mathrm{y}-\mathrm{y}_{\mathrm{s}}}{\mathrm{y}_{\mathrm{w}}}=\mathrm{x}, \mathrm{dy}=\mathrm{y}_{\mathrm{w}} \mathrm{dx}$ in equation (9) we have:

$$
\begin{aligned}
& \sim \exp \left(-\left(\sigma \sqrt{y_{s}}\right)^{2}\right)\left[y_{s}^{(\lambda-1)}-y_{w} x y_{s}{ }^{(\lambda-2)}\left(-\lambda+\sigma^{2} y_{s}+1\right)+\right. \\
& \frac{\left(y_{w} x\right)^{2}}{2} y_{s}^{(\lambda-3)}\left(\sigma^{4} y_{s}^{2}+2(1-\lambda) \sigma^{2} y_{s}+\left(\lambda^{2}-3 \lambda+2\right)\right)- \\
& \frac{\left(y_{w} x\right)^{3}}{6} y_{s}{ }^{(\lambda-4)}\left(\sigma^{6} y_{s}^{3}+3(1-\lambda) \sigma^{4} y_{s}^{2}+\left(3 \lambda^{2}-9 \lambda+6\right) \sigma^{2} y_{s}-\right. \\
& \left.\left.\lambda^{3}+6 \lambda^{2}-11 \lambda+6\right)\right] \\
& 1-X \sim \\
& \frac{\alpha}{\Gamma(\lambda)} \int_{0}^{\infty}(\exp (-\exp (-x))-H(x)) \exp \left(-\left(\sigma \sqrt{y_{s}}\right)^{2}\right)\left[y_{s}^{(\lambda-1)}-\right. \\
& y_{w} x \mathrm{y}_{\mathrm{s}}{ }^{(\lambda-2)}\left(-\lambda+\sigma^{2} y_{s}+1\right)+\frac{\left(y_{w} x\right)^{2}}{2} y_{s}{ }^{(\lambda-3)}\left(\sigma^{4} y_{s}{ }^{2}+\right. \\
& \left.2(1-\lambda) \sigma^{2} y_{s}+\left(\lambda^{2}-3 \lambda+2\right)\right)-\frac{\left(y_{w} x\right)^{3}}{6} y_{s}^{(\lambda-4)}\left(\sigma^{6} y_{s}^{3}+\right. \\
& 3(1-\lambda) \sigma^{4} y_{s}{ }^{2}+\left(3 \lambda^{2}-9 \lambda+6\right) \sigma^{2} y_{s}-\lambda^{3}+6 \lambda^{2}-11 \lambda+ \\
& \text { 6) }] y_{w} d x+\frac{\alpha}{\sigma^{2 \lambda} \Gamma(\lambda)} \Gamma\left(\lambda, \sigma^{2} y_{s}\right)
\end{aligned}
$$

or

$$
\begin{aligned}
& 1-X \sim \frac{\alpha}{\Gamma(\lambda)} \exp \left(-\left(\sigma \sqrt{y_{s}}\right)^{2}\right) y_{s}^{(\lambda-1)} y_{w}\left[L_{0}-\frac{y_{w}}{y_{s}} L_{1}\left(-\lambda+\sigma^{2} y_{s}+\right.\right. \\
& 1)+\frac{1}{2}\left(\frac{y_{w}}{y_{s}}\right)^{2} L_{2}\left(\sigma^{4} y_{s}^{2}+2(1-\lambda) \sigma^{2} y_{s}+\left(\lambda^{2}-3 \lambda+2\right)\right)-
\end{aligned}
$$


$\frac{1}{6}\left(\frac{y_{w}}{y_{s}}\right)^{3} L_{3}\left(\sigma^{6} y_{s}^{3}+3(1-\lambda) \sigma^{4} y_{s}^{2}+\left(3 \lambda^{2}-9 \lambda+6\right) \sigma^{2} y_{s}-\right.$

$\left.\left.\lambda^{3}+6 \lambda^{2}-11 \lambda+6\right)\right]+\frac{\alpha}{\sigma^{2 \lambda} \Gamma(\lambda)} \Gamma\left(\lambda, \sigma^{2} y_{s}\right)$

We know that:

$\frac{\Gamma\left(\lambda, \sigma^{2} y_{s}\right)}{\Gamma(\lambda)}=1-P\left(\lambda, \sigma^{2} y_{s}\right)$

where $P\left(\lambda, \sigma^{2} y_{s}\right)=\frac{\gamma\left(\lambda, \sigma^{2} y_{s}\right)}{\Gamma(\lambda)}$ is the lower cumulative distribution for Gamma random variables and $\gamma\left(\lambda, \sigma^{2} y_{s}\right)$ is the lower incomplete gamma function.

$L_{0} \approx-0.5772, L_{1} \approx-0.98906, L_{2} \approx-1.81496, L_{3}$ $\approx-5.89037$

The remaining integral terms are evaluated by the expression: $L_{n} \equiv \int_{-\infty}^{\infty} x^{n}\left(e^{-e^{-x}}-H(x)\right) d x$

\section{Case 2: For $\mathrm{n}^{\text {th }}$ order reaction $(\mathbf{n} \neq \mathbf{1})$}

Invoking the equation (3), we can derive the result for $\mathrm{n}^{\text {th }}$ order reaction. As stated earlier, the term $\left(\frac{E}{R \ominus t}\right) \rightarrow \infty$, the exponential term raised to power $\left(\frac{1}{1-n}\right)$, will vary from zero to one, and is approximated by using the binomial expansion. Hence, the equation (3) is written as:

$(1-X)_{n^{t h}} \sim \int_{0}^{\infty}\left[1-\exp \left(\frac{E_{s}-E}{E_{w}}\right)+\frac{n}{2} \exp \left(2\left(\frac{E_{s}-E}{E_{w}}\right)\right)-\right.$

$\left.\frac{(2 n-1)}{6} \exp \left(3\left(\frac{E_{s}-E}{E_{w}}\right)\right)+\cdots\right] \frac{E^{(\lambda-1)} e^{-\frac{E}{\eta}}}{\eta^{\lambda} \Gamma(\lambda)} d E$

$(1-X)_{n^{t h}} \sim \int_{0}^{\infty}\left[1-\exp \left(\frac{y_{s}-y}{y_{w}}\right)+\frac{n}{2} \exp \left(2\left(\frac{y_{s}-y}{y_{w}}\right)\right)-\right.$

$\left.\frac{(2 n-1)}{6} \exp \left(3\left(\frac{y_{s}-y}{y_{w}}\right)\right)+\cdots\right] \frac{\alpha y^{(\lambda-1)} \exp \left(-(\sigma \sqrt{y})^{2}\right)}{\Gamma(\lambda)} d y$

After applying the wide distribution limit, the above equation is expressed in the form of Heaviside or unit step function as:

$(1-X)_{n^{\text {th }}} \sim \int_{0}^{\infty}\left[1-\left(\exp \left(\frac{y_{s}-y}{y_{w}}\right)-H\left(y_{s}-y\right)\right)+\right.$

$\frac{n}{2}\left(\exp \left(2\left(\frac{y_{s}-y}{y_{w}}\right)\right)-H\left(y_{s}-y\right)\right)-\frac{(2 n-1)}{6}\left(\exp \left(3\left(\frac{y_{s}-y}{y_{w}}\right)\right)-\right.$

$\left.\left.-H\left(y_{s}-y\right)\right)+\cdots\right] \frac{\alpha y^{(\lambda-1)} \exp \left(-(\sigma \sqrt{y})^{2}\right)}{\Gamma(\lambda)} d y$

or:

$(1-X)_{n^{\text {th }}} \sim \frac{\alpha}{\sigma^{2 \lambda}}\left(1+\frac{(n-5)}{6} \frac{\Gamma\left(\lambda, \sigma^{2} y_{s}\right)}{\Gamma(\lambda)}\right)+$

$\frac{\alpha}{\Gamma(\lambda)} \exp \left(-\left(\sigma \sqrt{y_{s}}\right)^{2}\right) y_{s}^{(\lambda-1)} y_{w}\left(\left[\left(P_{0}+\frac{n}{2} M_{0}-\frac{(2 n-1)}{6} N_{0}\right)-\right.\right.$

$\frac{y_{w}}{y_{s}}\left(P_{1}+\frac{n}{2} M_{0}-\frac{(2 n-1)}{6} N_{1}\right)\left(-\lambda+\sigma^{2} y_{s}+1\right)+\frac{1}{2}\left(\frac{y_{w}}{y_{s}}\right)^{2}\left(P_{2}+\right.$

$\left.\frac{n}{2} M_{2}-\frac{(2 n-1)}{6} N_{2}\right)\left(\sigma^{4} y_{s}^{2}+2(1-\lambda) \sigma^{2} y_{s}+\left(\lambda^{2}-3 \lambda+2\right)\right)-$

$\frac{1}{6}\left(\frac{y_{w}}{y_{s}}\right)^{3}\left(P_{3}+\frac{n}{2} M_{3}-\frac{(2 n-1)}{6} N_{3}\right)\left(\sigma^{6} y_{s}{ }^{3}+3(1-\lambda) \sigma^{4} y_{s}{ }^{2}+\right.$

$\left.\left.\left.\left(3 \lambda^{2}-9 \lambda+6\right) \sigma^{2} y_{s}-\lambda^{3}+6 \lambda^{2}-11 \lambda+6\right)\right]\right)$

\subsubsection{Erlang Distribution (Generalized Chi-squared distribution) (for the positive integer value of $\lambda>0$ )}

\section{Case 1: First order reaction}

For Erlang distribution, we can rewrite the equation (11) as: $1-X \sim \frac{\alpha}{(\lambda-1) !} \exp \left(-\left(\sigma \sqrt{y_{s}}\right)^{2}\right) y_{s}^{(\lambda-1)} y_{w}\left[L_{0}-\frac{y_{w}}{y_{s}} L_{1}(-\lambda+\right.$ $\left.\sigma^{2} y_{s}+1\right)+\frac{1}{2}\left(\frac{y_{w}}{y_{s}}\right)^{2} \mathrm{~L}_{2}\left(\sigma^{4} y_{s}^{2}+2(1-\lambda) \sigma^{2} y_{s}+\left(\lambda^{2}-3 \lambda+\right.\right.$ 2)) $-\frac{1}{6}\left(\frac{y_{w}}{y_{s}}\right)^{3} L_{3}\left(\sigma^{6} y_{s}{ }^{3}+3(1-\lambda) \sigma^{4} y_{s}^{2}+\left(3 \lambda^{2}-9 \lambda+\right.\right.$ 6) $\left.\left.\sigma^{2} y_{s}-\lambda^{3}+6 \lambda^{2}-11 \lambda+6\right)\right]+\frac{\alpha}{\left.\sigma^{2 \lambda}(\lambda-1) !\right)} \Gamma\left(\lambda, \sigma^{2} y_{s}\right)$

In case of the Erlang distribution, we have:

$\frac{\Gamma\left(\lambda, \sigma^{2} y_{s}\right)}{(\lambda-1) !}=\operatorname{Pr}\left(\lambda, \sigma^{2} y_{s}\right) \approx 1-\sum_{f=0}^{\lambda-1} \frac{1}{f !} e^{-\left(y_{s} \sigma^{2}\right)}\left(\sigma^{2} y_{s}\right)^{f}$

where $\operatorname{Pr}\left(\lambda, \sigma^{2} \mathrm{y}_{\mathrm{s}}\right)$ is the cumulative distribution for the Poisson random variable.

\section{Case 2: $\mathrm{n}^{\text {th }}$ order reaction}

The $\mathrm{n}^{\text {th }}$ order reaction case of the Erlang distribution is expressed as:

$(1-X)_{n^{t h}} \sim \frac{\alpha}{\sigma^{2 \lambda}}\left(1+\frac{(n-5)}{6} \frac{\Gamma\left(\lambda, \sigma^{2} y_{s}\right)}{(\lambda-1) !}\right)+$ $\frac{\alpha}{(\lambda-1) !} \exp \left(-\left(\sigma \sqrt{y_{s}}\right)^{2}\right) y_{s}^{(\lambda-1)} y_{w}\left(\left[\left(P_{0}+\frac{n}{2} M_{0}-\frac{(2 n-1)}{6} N_{0}\right)-\right.\right.$ $\frac{y_{w}}{y_{s}}\left(P_{1}+\frac{n}{2} M_{0}-\frac{(2 n-1)}{6} N_{1}\right)\left(-\lambda+\sigma^{2} y_{s}+1\right)+\frac{1}{2}\left(\frac{y_{w}}{y_{s}}\right)^{2}\left(P_{2}+\right.$ $\left.\frac{n}{2} M_{2}-\frac{(2 n-1)}{6} N_{2}\right)\left(\sigma^{4} y_{s}^{2}+2(1-\lambda) \sigma^{2} y_{s}+\left(\lambda^{2}-3 \lambda+2\right)\right)-$ $\frac{1}{6}\left(\frac{y_{w}}{y_{s}}\right)^{3}\left(P_{3}+\frac{n}{2} M_{3}-\frac{(2 n-1)}{6} N_{3}\right)\left(\sigma^{6} y_{s}^{3}+3(1-\lambda) \sigma^{4} y_{s}^{2}+\right.$ $\left.\left.\left.\left(3 \lambda^{2}-9 \lambda+6\right) \sigma^{2} y_{s}-\lambda^{3}+6 \lambda^{2}-11 \lambda+6\right)\right]\right)$

The values of coefficients are estimated as:

$P_{0} \approx-0.36788, P_{1} \approx-0.23576, P_{2} \approx-0.17273, P_{3} \approx$ -0.13607 ,

$M_{0} \approx-0.56767, M_{1} \approx-0.35150, M_{2} \approx-0.25250, M_{3} \approx$ -0.19642 ,

$N_{0} \approx-0.68326, N_{1} \approx-0.41102, N_{2} \approx-0.29061, N_{3} \approx$ -0.22387 .

The remaining integral terms are evaluated as:

$$
\begin{aligned}
& P_{n} \equiv \int_{-\infty}^{\infty} x^{i}(\exp (-x)-U(x)) d x, i=0,1,2,3 \ldots \\
& M_{n} \equiv \int_{-\infty}^{\infty} x^{i}(\exp (-2 x)-U(x)) d x, i=0,1,2,3 \ldots \\
& N_{n} \equiv \int_{-\infty}^{\infty} x^{i}(\exp (-3 x)-U(x)) d x, i=0,1,2,3 \ldots
\end{aligned}
$$




\section{APPLICATION OF THE FOREST WASTE}

For application perspective, the thermogravimetric analysis has been performed upon the sample of pine needles. The date of elemental composition and the calorific value have been obtained by using the CHNO-S analyser, and the bomb calorimeter, whereas the thermal degradation of the same sample has been carried out under the presence of inert atmosphere of nitrogen, and with the help of TG/DTG (SDT Q600 (TA, Perkin Elmer)) analyser. The range of temperature varied from $292 \mathrm{~K}$ to $873 \mathrm{~K}$ in the furnace. The thermocouple type ' $R$ ' has been used to measure the sample and the furnace temperature. A vertical TG/DCS holder was used to hold the sample. To perform pyrolsis, a nitrogen purge flow rate was set at $100 \mathrm{ml} / \mathrm{min}$, whose function is to remove the product gases. Thermogravimetric measurements are done at the heating rate of $10{ }^{\circ} \mathrm{C} / \mathrm{min}$. $\mathrm{Al}_{2} \mathrm{O}_{3}$ crucibles are used. The furnace space is inertized in order to eliminate the remaining oxygen. The mass of the samples is in between $20 \mathrm{mg}$ to $21 \mathrm{mg}$.

Thermogravimetric data has used for the prediction of nthorder DAEM using the Gamma distribution (Fig. 8). Equations (11), (12), (13), and (14) are solved by using algorithm on the Matlab software. Each parameter is compared and reiterated until the root mean square error between experimental and simulated values is not less than equal to the maximum permissible error.

Tab. 1. Elemental composition and the high heating value (H.H.V) of pine needle sample

\begin{tabular}{|c|c|c|c|c|c|c|c|}
\hline $\mathrm{C} \%$ & $\mathrm{H} \%$ & $\mathrm{~N} \%$ & $\mathrm{O} \%$ & $\mathrm{~S} \%$ & Ash\% & $\begin{array}{c}{ }^{*} \mathrm{H} . \mathrm{H} . \mathrm{V} \\
(\mathrm{kJ} / \mathrm{kg})\end{array}$ & $\begin{array}{c}{ }^{*} \mathrm{~V} . \mathrm{M} \\
\%\end{array}$ \\
\hline 53.7 & 5.21 & 0.61 & 32.13 & 0.22 & 4.72 & 19.5 & 70.1 \\
\hline
\end{tabular}

${ }^{*} \mathrm{H} . \mathrm{H}$. V- Higher heating value

${ }^{*} \mathrm{~V} . \mathrm{M}$ - Volatile matter

Tab. 2. Comparative illustration of Gamma and Erlang distributions with other distribution types

\begin{tabular}{|c|c|c|}
\hline Distribution Types & $\begin{array}{c}\text { Upper limit of ' } d E \text { ' } \\
\text { integrals }\end{array}$ & $\begin{array}{c}\text { Frequency factor } \\
\left(\mathbf{m i n}^{-1}\right)\end{array}$ \\
\hline $\begin{array}{c}\text { Gaussian } \\
\text { (Dhaundiyal and } \\
\text { Singh, 20016) }\end{array}$ & $80 \mathrm{~kJ} / \mathrm{mol}$ & $1.75 \mathrm{E}+06$ \\
\hline $\begin{array}{c}\text { Weibull } \\
\text { (Dhaundiyal and } \\
\text { Singh ,2016) }\end{array}$ & $23 \mathrm{~kJ} / \mathrm{mol}$ & $10 \mathrm{E}+03$ \\
\hline Gamma & $150 \mathrm{~kJ} / \mathrm{mol}$ & 0.0015 \\
\hline Erlang & $150 \mathrm{~kJ} / \mathrm{mol}$ & 0.0015 \\
\hline
\end{tabular}

Tab. 3. Computed root mean square error

\begin{tabular}{|c|c|}
\hline Distribution function & Root mean squared error (RMSE) \\
\hline Gamma & $10 \mathrm{E}-02$ to $10 \mathrm{E}-03$ \\
\hline Erlang & $10 \mathrm{E}-02$ to $10 \mathrm{E}-03$ \\
\hline
\end{tabular}

$$
R M S E^{* *}=\sqrt{\frac{\sum_{i=1}^{N}\left(\left(1-X_{i}\right)_{\text {Experimental }}-\left(1-X_{i}\right)_{\text {Evaluated }}\right)}{N}}
$$

where $N$ is number of data points. a)

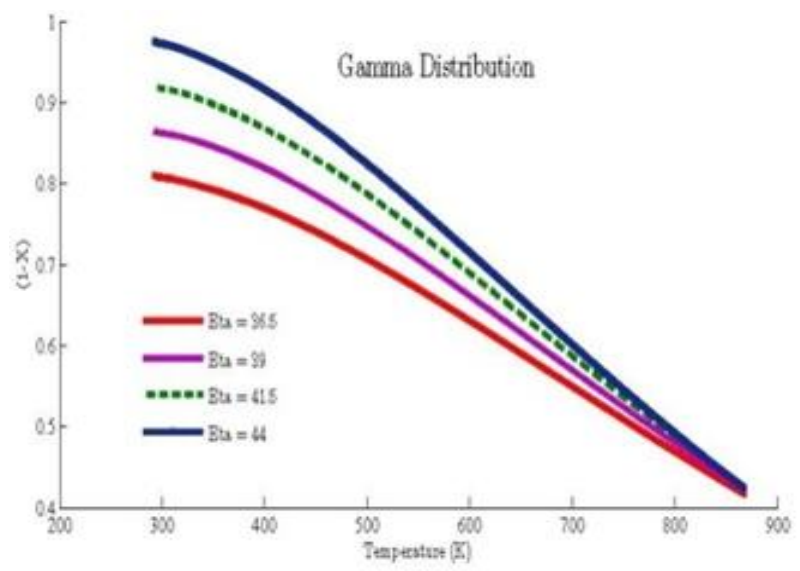

b)

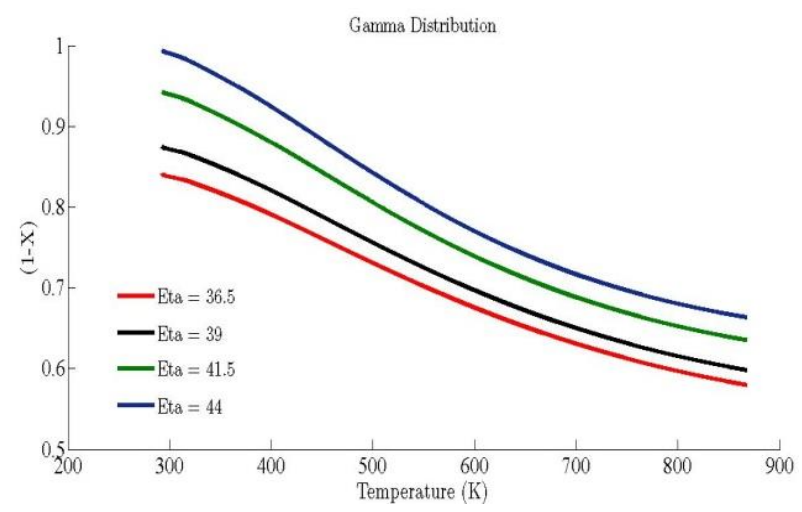

Fig. 1. Effect of shape parameter of the Gamma distribution $(\eta)$ on the numerical results $\left(k_{0}=5.7 \mathrm{E}-04 \mathrm{~min}^{-1}, \theta=10^{\circ} \mathrm{C} / \mathrm{min}\right.$, $T_{0}=293 \mathrm{~K}$ and $\lambda=45.5 \mathrm{~kJ} / \mathrm{mol}$; (a) first order reaction, (b) $\mathrm{n}^{\text {th }}$ order reaction, $n=8.5$ )

a)

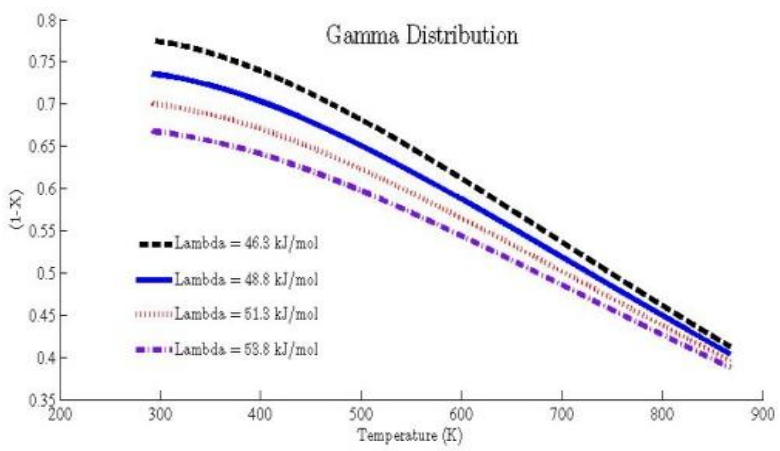

b)

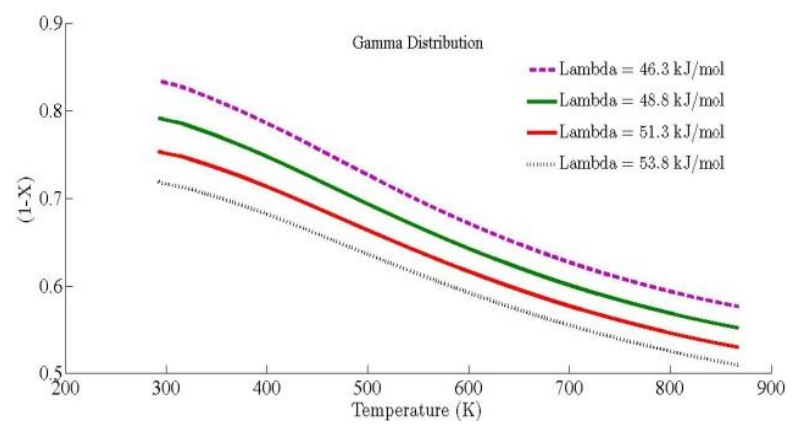

Fig. 2. Effect of scale parameter of the Gamma distribution $(\lambda)$ on the numerical results $\left(k_{0}=5.7 \mathrm{E}-04 \mathrm{~min}^{-1}, \theta=10^{\circ} \mathrm{C} / \mathrm{min}, T_{0}=293 \mathrm{~K}\right.$ and $\eta=35$; (a) first order, (b) $n^{\text {th }}$ order, $n=8.5$ ) 
a)

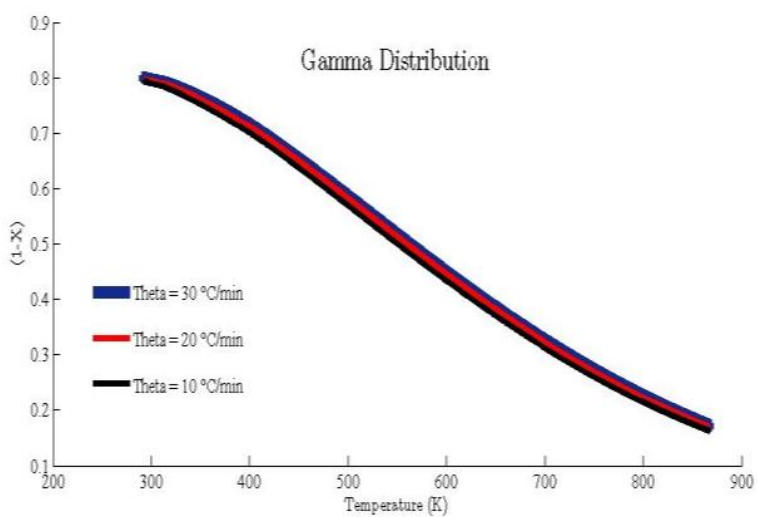

b)

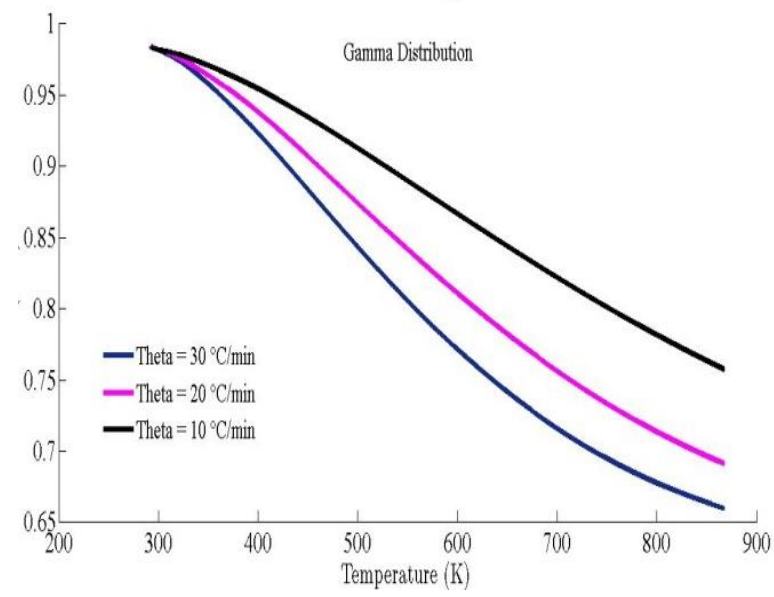

Fig. 3. Effect of heating rate on the numerical results $\left(k_{0}=5.7 \mathrm{E}-04 \mathrm{~min}^{-1}\right.$, $\lambda=45.5 \mathrm{~kJ} / \mathrm{mol}, T_{0}=293 \mathrm{~K}$ and $\eta=35$; (a) first order,

(b) $\mathrm{n}^{\text {th }}$ order, $n=8.5$ )

a)

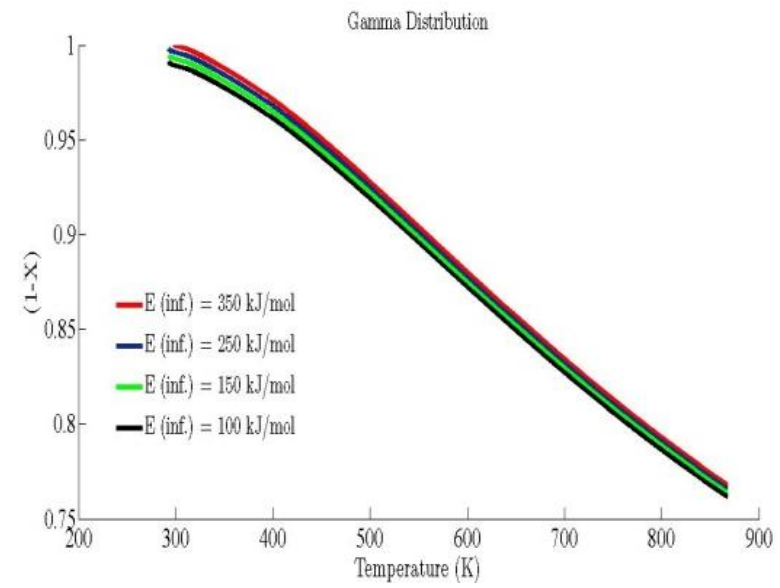

b)

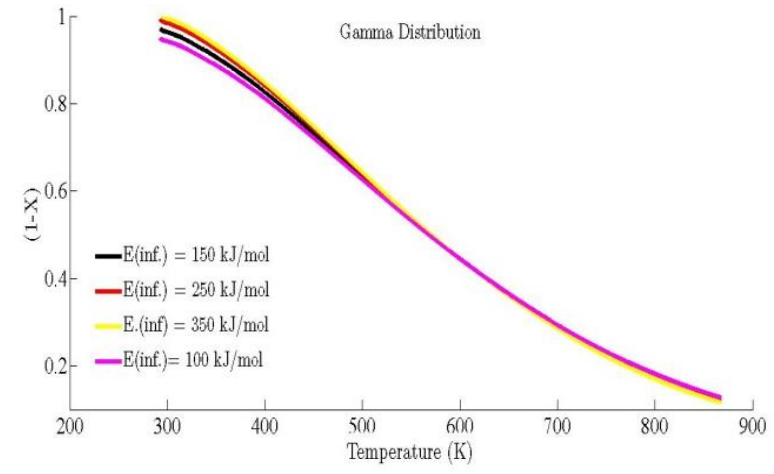

Fig. 4. Effect of upper limit $\left(E_{\infty}\right)$ of $d E$ integral on the numerical results $\left(k_{0}=5.7 \mathrm{E}-04 \mathrm{~min}^{-1}, \lambda=45.5 \mathrm{~kJ} / \mathrm{mol}, T_{0}=293 \mathrm{~K}\right.$ and $\eta=35$; (a) first order, (b) $\mathrm{n}^{\text {th }}$ order, $n=8.5$ ) a)

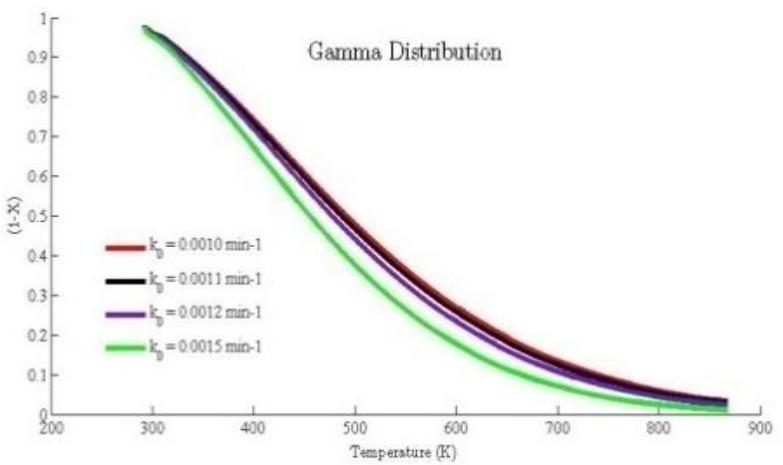

b)

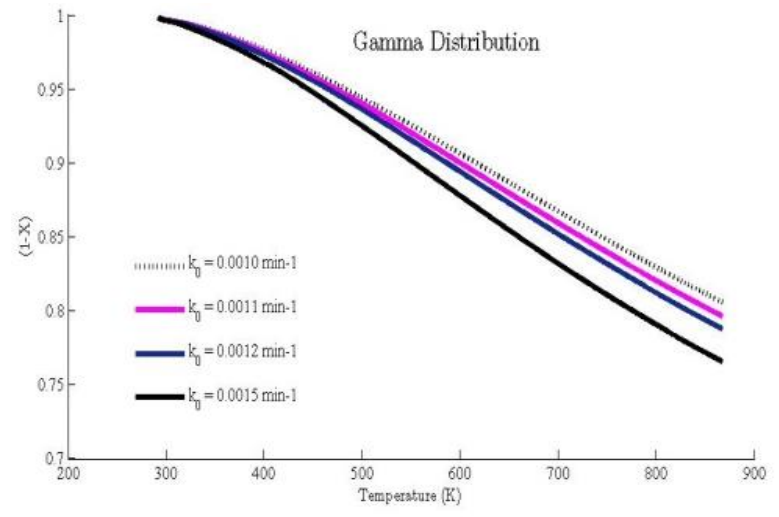

Fig. 5. Effect of frequency factor $\left(k_{0}\right)$ on the numerical results ( $\lambda=45.5 \mathrm{~kJ} / \mathrm{mol}, T_{0}=293 \mathrm{~K}$ and $\eta=35$; (a) first order, (b) $\mathrm{n}^{\text {th }}$ order, $n=8.5$ )

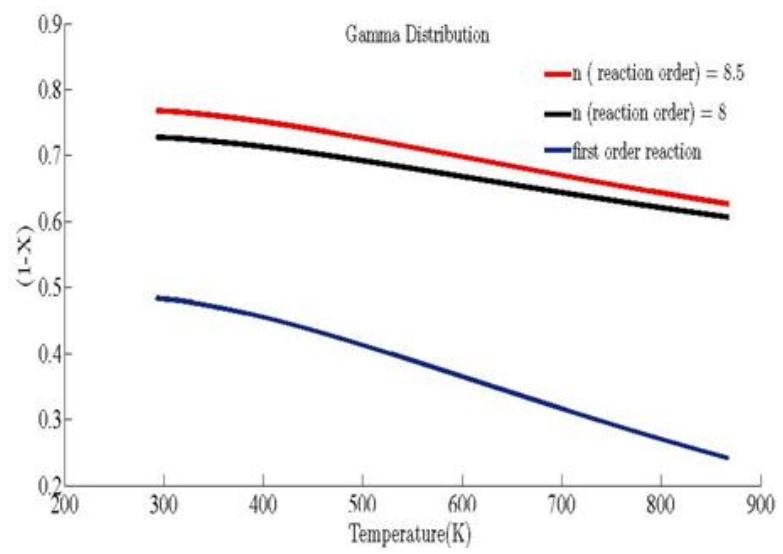

Fig. 6. Effect of reaction order $(n)$ on the numerical results $\left(\lambda=45.5 \mathrm{~kJ} / \mathrm{mol}, T_{0}=293 \mathrm{~K}\right.$; and $\left.\eta=35\right)$

a)

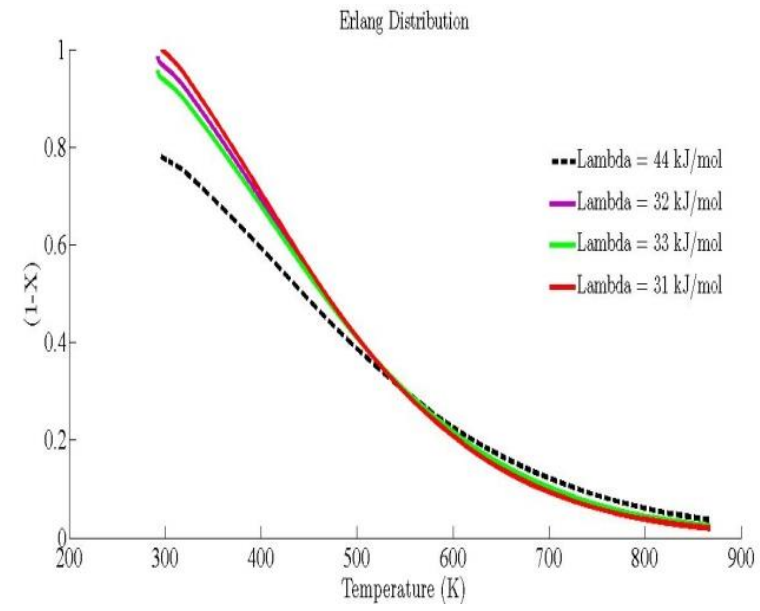


b)

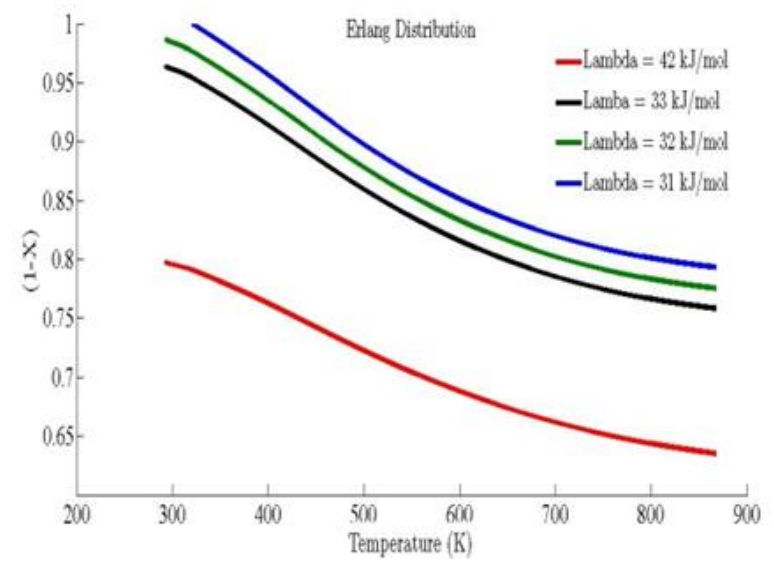

Fig. 7. Effect of scale parameter of the Erlang distribution $(\lambda)$ on the numerical results $\left(k_{0}=0.0047 \mathrm{~min}^{-1}, \theta=10^{\circ} \mathrm{C} / \mathrm{min}, T_{0}=293 \mathrm{~K}\right.$ and $\eta=32$; (a) first order, (b) $\mathrm{n}^{\text {th }}$ order, $n=6.8$ )

a)

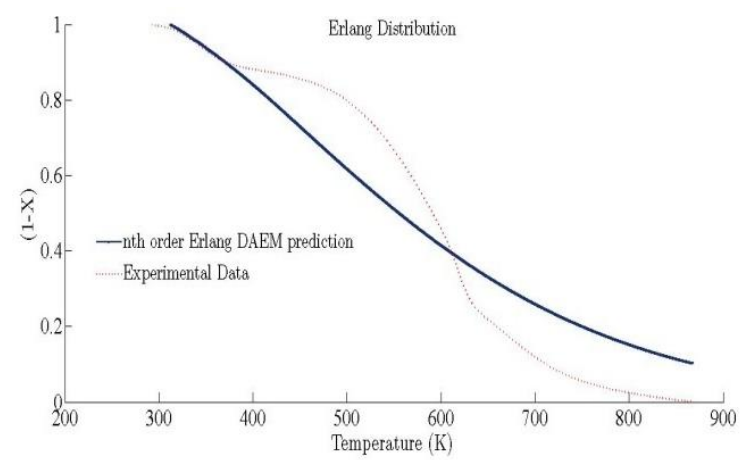

b)

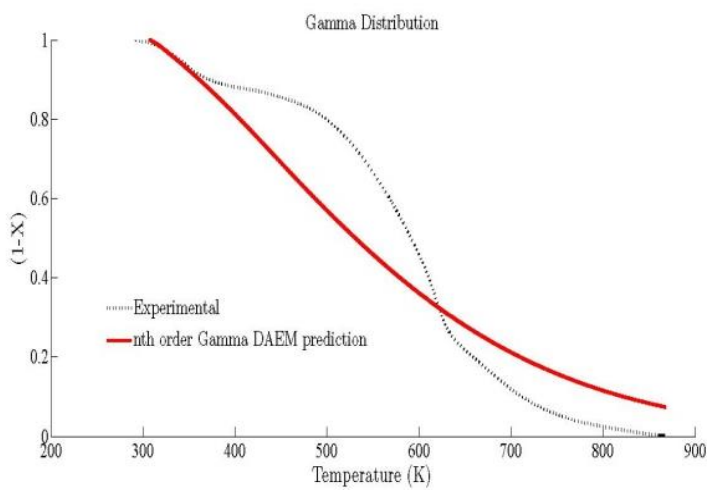

Fig. 8. Comparison between the experimental data and the $\mathrm{n}^{\text {th }}$ order DAEM prediction (a) The Erlang Distribution (b) The Gamma Distribution

\section{RESULTS AND DISCUSSION}

The shape parameter has been obtained for Gamma distribution by using the asymptotic approximation and the energy rescaling $(y)$ of activation energies. The influence of shape parameter of Gamma distribution on the numerical results is shown in Fig. 1. At the beginning of pyrolysis, the remaining mass fraction $(1-X)$ (equations 11 and 12) must be at vicinity of one. On the contrary, in Fig. 1, it is observed that the remaining mass fraction is less than one. However, as the value of shape parameter approaches $44 \leq \eta$, the upper half of curve approaches one. The distribution exhibits no inflexion for the lower value of shape parameter $39 \geq \eta$ and the remaining mass fraction curves lead towards right as parallel to each other. The effect of scale pa- rameter values $(\lambda)$ of Gamma distribution on the numerical result is shown in Fig. 2. The decrease in the value of scale parameter, $\lambda$, reduces the slope of the remaining mass proportion $(1-X)$ (equations 11and 12) curves. The effect of positive integer value of scale parameter of Erlang distribution is depicted in Fig. 7. It is observed that the behaviour of scale parameter $(\lambda)$ for Erlang distribution is opposite to that of Gamma distribution. As the value of $\lambda \leq 33$, the remaining mass fraction curve has proximity with one for the Erlang distribution, which implies the good approximation is obtained at the beginning of pyrolysis. The variation has been observed as the second term is almost negligible in equation (14), and the remaining mass fraction behaves like the cumulative distribution function. The effect of heating rate on numerical results is illustrated in Fig. 3, where it is seen that the remaining mass proportion is slightly increased with the heating rate. It has been observed that the mass fraction curves are shifted up the temperature scale with an increase in the heating rate. The influence of the outer limit of ' $\mathrm{d} E$ ' on the numerical results of the nonisothermal nth order DAEM is shown in Fig. 4. It reveals that remaining mass proportion is at vicinity of one for $E_{\infty} \leq$ $150 \mathrm{~J} / \mathrm{mol}$ values. When the values of $E$ are more than 250 $\mathrm{J} / \mathrm{mol}$, the results are not accurate and closely proximate to each other. Hence, $150 \mathrm{~J} / \mathrm{mol}$ is used as the upper limit of the $\mathrm{d} E$ integral. The effect of the frequency factor $\left(k_{0}\right)$ values on the numerical results is shown in Fig. 5. According to these curves, increase in $\mathrm{k} 0$ values causes $(1-\mathrm{X})$ (equations 11 and 12) curves to shift towards the left direction. The effect of the reaction order $(n)$ values on the numerical result is illustrated in Fig. 6 . As the order of reaction increases, the lower half range of $(1-X)$ (equations 11 and 12) curves shifted up. The prediction of nth order DAEM is depicted in Fig. 8. In the beginning of pyroylsis, Erlang distribution (Fig. 8a) and Gamma distribution (Fig. 8b) are in good agreement with the thermogravimetric data, but as time increases, the gamma distribution has relatively provided an accurate approximation for the conversion occurred at the higher temperature.

Variation in the upper limit of activation energy and frequency factors for the different distribution function is shown in Tab. 3. But, in case of the Gamma and the Erlang distributions, it was found that evaluation of kinetic parameters at single heating rate led to the compensatory effect (Doyle,1962). Root mean squared error between Erlang and Gamma distribution functions is illustrated in Tab. 3.

\section{CONCLUSIONS}

In the asymptotic approximation of the nonisothermal $\mathrm{n}^{\text {th }}$ order for knowing the influence of parametric values on the pyrolysis kinetics, $150 \mathrm{~J} / \mathrm{mol}$ value is used as the limit of the outer $\mathrm{dE}$ integral. The variation of the frequency factor, heating rate, the reaction order, the shape, and the scale parameter of the Gamma distribution as well as Erlang distribution affect the form of remaining mass fraction curves. We also studied the influence of some parameters. The special case of Gamma distribution has been discussed to know the more practical aspect regarding the distribution behaviour at lower and higher temperature regimes. It has been concluded that the Gamma distribution provides better approximation for conversion rate at higher temperature than that of the Erlang distribution. The Erlang distribution is found to be more suiTab. for pyrolysis reaction that took place for the lower 
temperature regimes. Moreover, the results are very helpful to determine the kinetic parameters of the nonisothermal $\mathrm{n}^{\text {th }}$ order Gamma DAEM from the thermo analytical data of biomass pyolysis. It can also be concluded from comparative illustration that the two asymmetrical distribution functions (Weibull and Gamma) does not provide the same results (Tab. 3.)

\section{REFERENCES}

1. Anthony D.B. (1974), Rapid devolatilization and hydrogasification of pulverized coal, DSc. thesis, Massachusetts Institute of Technology.

2. Armstrong R., Kulesza B.L.J. (1981), An approximate solution to the equation $x=\exp (-x / \epsilon)$, Bull. Institute of Mathematics and its Applications, 17, 56.

3. Brown M. E. (2001), Introduction to Thermal Analysis, Techniques and Applications, Kluwer Academic Publisher, Dordrecht.

4. Burnham A.K., Braun R.L. (1999),Global kinetic analysis of complex materials, Energy Fuels, 13, 1-22.

5. Burnham A.K., Schmidt B.J., and Braun R.L (1995), A test of parallel reaction model using kinetic measurements on hydrous pyrolysis residues, Geochem, 23, 931-939.

6. Capart R, Khezami L., Burnham A.K. (2004), Assessment of various kinetic models for the pyrolysis of microgranular cellulose, Thermochim. Acta, 417(1), 79-89.

7. Conesa J. A., Marcilla A., Caballero J. A., Font R. (2001), Comments on the validity and utility of the different methods for kinetic analysis of thermogravimetric data, J. Anal. Appl. Pyrolysis, $617,58-59$.

8. Conesa J.A., Caballero J.A., Marcilla A., Font R. (1995), Analysis of different kinetic models in the dynamic pyrolysis of cellulose, Thermochim. Acta, 254, 175-192.

9. Criado J.M., Pérez-Maqueda L.A. (2005), Sample controlled thermal analysis and kinetics, J. Therm. Anal. Cal., 80, 27-33.

10. Dhaundiyal A., Singh S.B. (2016), Asymptotic approximations to the distributed activation energy model for non-isothermal pyrolysis of loose biomass using the Weibull distribution, Archivum Combustionis, 36(2), 131-146.

11. Dhaundiyal A., Singh S.B. (2016), Proceedings of the Latvian Academy of Sciences, Section B. Natural, Exact, and Applied Sciences, 70, 64-70.

12. Di Blasi C. (2008), Modeling chemical and physical processes of wood and biomass pyrolysis, Progress in Energy and Combustion Science, 34, 47-90.

13. Doyle C.D. (1962), Estimating isothermal life from thermogravimetric data, J. Appl. Polym. Sci. 6, 639-642.

14. Ferdous D, Dalai A.K, Bej S.K., Thring R.W. (2002), Pyrolysis of lignins, experimental and kinetics studies, Energy Fuels, 16 $1405-1412$

15. Folgueras M.B., Diaz R.M., Xiberta J., Prieto I. (2003), Thermogravimetric analysis of the co-combustion of coal and sewage sludge, Fuel, 82, 2051-2055.

16. Galgano A., Blasi C.D. (2003), Modeling wood degradation by the unreacted-core-shrinking approximation, Eng. Chem. Res, 42, 2101 2111.

17. Giuntoli J., de Jong W., Arvelakis S., Spliethoff H., Verkooijen A.H.M. (2009), Quantitative and kinetic TG-FTIR study of biomass residue pyrolysis, Dry distiller's grains with solubles (DDGS) and chicken manure, Journal of Analytical and Applied Pyrolysis, 85(1), 301-312.

18. Hanbaba P., van Heek K.H, Jüntgen H., Peters W. (1968), Nonisothermal kinetics of coal pyrol-yse , Part II , Extension of the theory of the evolution of gas and experimental confirmation of bituminous coal, Fuel Chemistry, 49(12), 1968, 368-376.,

19. Howard J.B. (1981), Fundamentals of Coal Pyrolysis and Hydropyrolysis, in Chemistry of Coal Utilization, (M.A.Elliott, Ed) Wiley \& Sons.
20. Koreňová Z., JumaM., Annus J., Markoš J., Jelemensky L. (2006), Kinetics of pyrolysis and properties of carbon black from a scrap tire, Chemical Papers, 60, 422-426.

21. Lakshmanan C.C., White N. (1994), A new distributed activation energy model using Weibull distribution for the representation of complex kinetics, Energy Fuels, 8, 1158-1167.

22. Lapuerta, M., Hernández, J.J., Rodríguez, J. (2004), Kinetics of devolatilisation of forestry wastes from thermogravimetric analysis, Biomass and Bioenergy, 27(4),385-91.

23. Mysyk R.D., Whyman G.E., Savoskin M.V., Yaroshenko A.P. (2005), Theoretical model and experimental study of pore growth during thermal expansion of graphite intercalation compounds, J. Therm. Anal and Cal., 79(3), 515-519.

24. Niksa S., Lau, C-W. (1993), Global rates of devolatilization for various coal types Combust, Flame, 94, 293-307

25. Otero M., Calvo L.F., Gil M.V., García A.I., Morán A. (2008), Cocombustion Of Different Sewage Sludge and Coal, A nonisothermal thermogravimetric kinetic analysis, Bioresource Technology, 99, 6311-19.

26. Pitt G.J. (1962), The kinetics of the evolution of volatile products from coal, Fuel, 1, 267-274

27. Pysiak J.J., Badwi Y.Al. (2004), Kinetic equations for thermal dissociation processes, 76, 521-528

28. Quan C., Li A., Gao N. (2009), Thermogravimetric analysis and kinetic study on large particles of printed circuit board wastes, Waste Management, 29, 2353-2360.

29. Robeva R., Davies R., Hodge T., Enyedi A. (2010), Mathematical Biology Modules Based on Modern Molecular Biology and Modern Discrete Mathematics, CBE Life Sciences Education (The American Society for Cell Biology), 9 (3), 227-240.

30. Skrdla P.J., Roberson R.T. (2005), Semiempirical equations for modeling solid-state kinetics based on a Maxwell-Boltzmann distribution of activation energies, applications to a polymorphic transformation under crystallization slurry conditions and to the thermal decomposition of AgMnO4 crystals, J. Phys. Chem. B, 109, 10611- 10619.

31. Solomon P.R., Hamblen D.G. (1983), Finding Order in Coal Pyrolysis Kinetics, Topical Report Submitted to the U.S. Department of Energy. Progr. Energy Combust. Sci., 9, 323-361.

32. Suuberg E.M. (1983), Approximate solution technique for nonisothermal, Gaussian distributed activation energy models, Combust. Flame, 50,243-245

33. Szczodrak J., Fiedurek J. (1996), Technology for conversion of lignocellulosic biomass to ethanol, Biomass and Bioenergy, 34, 367375.

34. Teng H., Hsieh C.T. (1999), Influence of surface characteristics on liquid-phase adsorption of phenol by activated carbons prepared from bituminous coa, Ind. Engg. Chem. Res, 37, 3618-3624.

35. Vand V. (1943), A theory of the irreversible electrical resistance changes of metallic films evaporated in vacuum, Proc. Phys. Soc.,55, 222-246

36. White J.E., Catallo W.J., Legendre B.L. (2011), Biomass pyrolysis kinetics, A comparative critical review with relevant agriculture residue case studies, J. Anal. Appl. Pyrolysis, 91 (1), 1-33,

37. Zhu H.M., Yan J.H., Jiang X.G., Lai Y.E., Cen K.F(2009), Analysis Of Volatile Species Kinetics During Typical Medical Waste Materials Pyrolysis Using A Distributed Activation Energy Model, Journal of Hazardous Materials, 162(2), 646-651. 